\title{
Diagnosis and management of malignant sublingual gland tumors: a narrative review
}

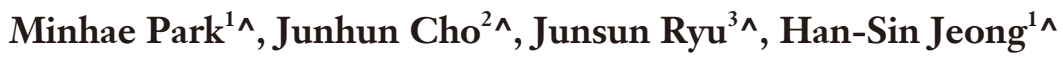 \\ ${ }^{1}$ Department of Otorhinolaryngology-Head and Neck Surgery, Seoul, Republic of Korea; ${ }^{2}$ Department of Pathology, Samsung Medical Center, \\ Sungkyunkwan University School of Medicine, Seoul, Republic of Korea; ${ }^{3}$ Head and Neck Oncology Clinic, National Cancer Center of Korea, Ilsan, \\ Republic of Korea \\ Contributions: (I) Conception and design: HS Jeong; (II) Administrative support: None; (III) Provision of study materials or patients: M Park, J Cho; \\ (IV) Collection and assembly of data: M Park, J Cho, J Ryu; (V) Data analysis and interpretation: M Park, J Cho, J Ryu; (VI) Manuscript writing: All \\ authors; (VII) Final approval of manuscript: All authors. \\ Correspondence to: Han-Sin Jeong, MD, PhD. Professor, Department of Otorhinolaryngology-Head and Neck Surgery, Samsung Medical Center, \\ Sungkyunkwan University School of Medicine, Seoul, Republic of Korea. Email: hansin.jeong@samsung.com.
}

Objective: In this article we review the literature on the malignant sublingual gland tumors from a surgical perspective.

Background Sublingual gland tumors occur with a very low incidence and most are malignant tumors. The extent of treatment, clinical outcomes and prognosis of malignant sublingual gland tumors have not been well defined, due to the rarity of this disease.

Methods: A database search using Web of Science (https://webofknowledge.com/) and PubMed (https:// pubmed.ncbi.nlm.nih.gov/) was conducted. The following keywords were used in the search: "sublingual gland tumor", AND "malignancy".

Conclusions: Although the sublingual glands are among the major salivary glands, they share common anatomical characteristics with minor salivary gland tumors. Therefore, the tumors from the sublingual gland may have different clinical behaviors from the other major salivary gland tumors. The sublingual glands are small without a true surrounding capsule of the glands; the extra-parenchymal extension is very common in sublingual gland tumors. Furthermore, the sublingual glands are located in close proximity to the lingual nerve, the submandibular gland-duct system and the mandible. Thus, the surgical approach to the malignant sublingual gland tumor should include the adequate management of neighboring structures. In addition, adjuvant radiation therapy provides a survival benefit for patients with malignant sublingual gland tumors, which have adverse features. This article summarizes the clinical characteristics and unique features of malignant sublingual gland tumors based on previous reports, and provides clinical information regarding the sublingual gland tumors to increase awareness of primary physicians as well as patients.

Keywords: Salivary gland neoplasms; sublingual gland; pathology; neoplasm invasiveness; treatment outcome

Submitted Sep 07, 2021. Accepted for publication Nov 05, 2021.

doi: 10.21037 /gs-21-620

View this article at: https://dx.doi.org/10.21037/gs-21-620

\footnotetext{
^ ORCID: Minhae Park, 0000-0002-0836-8913; Junhun Cho, 0000-0002-6089-9340; Junsun Ryu, 0000-0002-6730-6755; Han-Sin Jeong, 0000-0003-4652-0573.
} 


\section{Introduction}

Tumors arising from the sublingual gland in the floor of the mouth are uncommon, comprising less than $1.0 \%$ of all salivary gland tumors (1-6). Mass of the sublingual gland may be misdiagnosed as the more common diseases on the floor of the mouth, including sialolithiasis as well as inflammatory or chronic sialadenitis (7). Therefore, a timely diagnosis and treatment cannot be achieved, unless the clinicians have some knowledge regarding sublingual gland tumors or suspect them.

Unfortunately, a large proportion of sublingual tumors are malignant tumors (70-90\%) (3,5,8-10). In addition, the extent of treatment, clinical outcomes and prognosis of malignant sublingual gland tumors have not been well defined, due to the low incidence of this disease (11-14). In the largest retrospective study using a database of the Surveillance, Epidemiology, and End Results program of the US National Cancer Institute, 210 patients with malignant sublingual gland tumors were analyzed (3). The results provided a clear overview of the clinical characteristics, prognostic factors, and treatment outcomes of this rare disease (3). Five-year treatment outcomes for malignant sublingual gland tumor patients showed approximately $69-83 \%$ survival; host factors, tumor stage at diagnosis, and treatment factors were found significant prognostic indicators.

In this article, we summarized the clinical characteristics and unique features of malignant sublingual gland tumors, based on several previous reports. Although the sublingual glands are among the major salivary glands, they share common anatomical characteristics with minor salivary gland tumors, but not with the parotid and submandibular glands. Therefore, the tumors from the sublingual gland may have different clinical behaviors from other major salivary gland tumors. Based on this background, the present review provides clinical information regarding the sublingual gland tumors to increase awareness of primary physicians as well as patients. We also present the following article in accordance with the Narrative Review reporting checklist (available at https://dx.doi.org/10.21037/gs-21-620).

\section{Methods}

A database search using the online databases Web of Science (https://webofknowledge.com/) and PubMed (https:// pubmed.ncbi.nlm.nih.gov/) was conducted in August 1, 2021. The following keywords were used in the search: "sublingual gland tumor", AND "malignancy". Articles in English published between January 1960 and July 2021 were searched.

\section{Results}

Initially, a total of 496 articles were found in the database. Then, the authors reviewed the retrieved articles (abstract or text) and excluded 444 articles not related to the topic of discussion. In addition, we also excluded 38 case reports, which had below five cases.

As a result, we found 14 key references (3,5-7,10-17) including two review articles $(8,18)$ (Table 1).

\section{Discussion}

\section{Anatomy of the sublingual glands}

The sublingual glands are the smallest of the major salivary glands, approximately one-fifth the size of the submandibular gland and weighing $2-4 \mathrm{~g}$. The dimension is approximately $4-6 \mathrm{~cm}$ in anteroposterior length and $2-4 \mathrm{~cm}$ in width. The glands lie in a submucosal plane within the anterior floor of the mouth, superior to the mylohyoid muscle and deep to the sublingual folds (8). There is no true fascial capsule surrounding the glands, which are only covered by oral mucosa.

The sublingual glands are drained by 8 to 20 small excretory ducts (the ducts of Rivinus) into the mucosa of the oral floor, or by a sublingual duct (the duct of Bartholin), which connects to the submandibular duct $(5,8,12)$. In this area, the postganglionic lingual nerve traverses to the anterior portion of the tongue through or close to the sublingual glands $(5,20)$. Thus, tumors arising from the sublingual glands involve the risk of invasion into these neighboring structures (Figure 1). Due to its anatomical proximity, submandibular gland excision has also been recommended during surgical resection of advanced malignant sublingual gland tumors (6). In several previous studies, the significance of lingual nerve involvement in malignant sublingual gland tumors, particularly adenoid cystic carcinomas, have also been addressed $(5,6,21)$.

\section{Clinical manifestations and symptoms of sublingual gland tumors}

The early stage of malignant sublingual gland tumors presents with symptoms of painless and non-ulcerative 
Table 1 Key references for this review



mass on the floor of the mouth (Figure 2). Sublingual gland tumors in the advanced stage may have invaded into neighboring structures, such as lingual nerve and submandibular gland. Therefore, patients with advanced cases can present numbness in the tongue due to lingual nerve paralysis $(6,18)$.

Tumor obstruction of the submandibular duct can lead to swelling of the affected submandibular gland, potentially resulting in a false diagnosis of the underlying disease. The tumor is also misdiagnosed as metastatic nodes. Fortunately, the tongue immobility and dysarthria due to tumor invasion of the hypoglossal nerve are less likely to occur, in malignant sublingual gland tumors (12).

\section{Diagnosis}

With bimanual palpation of the mass in the mouth floor, the initial imaging diagnostic work-ups include computed tomography (CT) or ultrasonography (US). If the results indicate malignancy, metastasis work-ups [whole-body positron emission tomography (PET) with CT, and/or chest $\mathrm{CT}$ ] can provide overall information regarding the systemic disease burden. The local disease extent is further evaluated using magnetic resonance imaging (MRI) if indicated. If sublingual gland tumors abut on the mandible or they are clinically fixed to the mandible, CT with bone algorithm has high diagnostic accuracy (over 90\%) to predict the pathological mandibular invasion $(22,23)$.

The palpable mass in the floor of the mouth is usually easily accessible and aspiration cytology, needle biopsy, incisional, or submucosal deep biopsy can be performed. Therefore, pretreatment pathological diagnosis is achieved in most sublingual gland tumors (approximately 90\%) (7). When the tumor is located in the posterior portion of the floor of the mouth, or in the inferior portion of the sublingual gland (deep to the submandibular duct), a trans- 


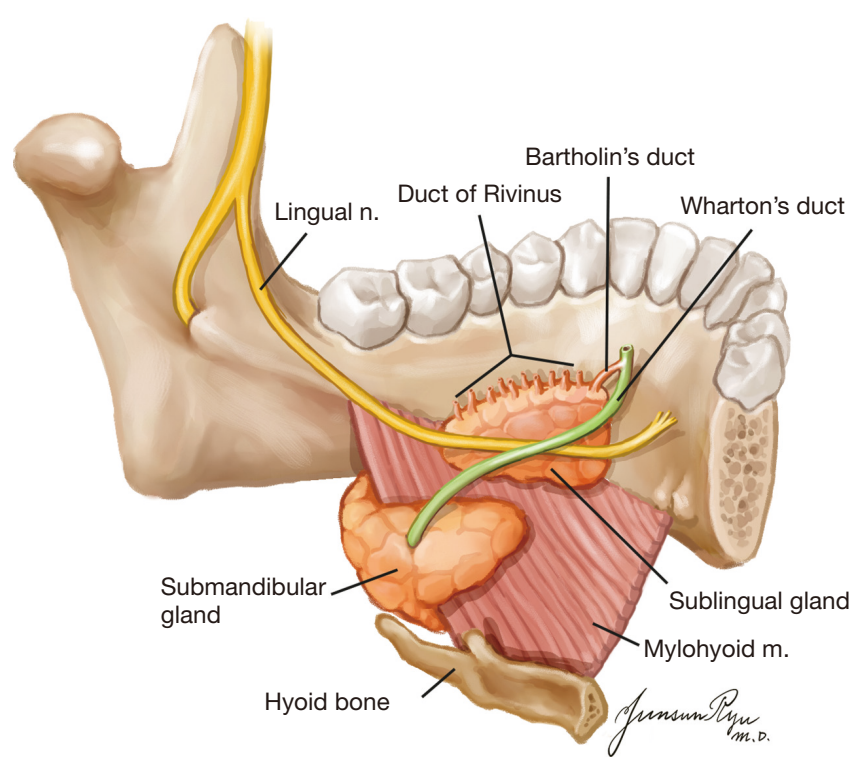

Figure 1 Anatomical relationship of sublingual gland and surrounding structures.

cervical US-guided fine-needle aspiration cytology is appropriate for diagnosis. In addition to the diagnosis of malignancy, the pathological subtypes can be accurately predicted in $75-80 \%$ of cases (7). In the remaining cases without a confirmative preoperative pathological diagnosis, a frozen section biopsy is helpful for diagnosis and decision of the surgical extent.

\section{Pathology}

Among the diverse pathological diagnoses of salivary glands, the two most common histological types of malignant sublingual gland tumors are mucoepidermoid carcinoma and adenoid cystic carcinoma, accounting for approximately $70 \%$ of malignant sublingual gland tumors $(3,5,7,8,10,15,17,18)$. The relative incidences and distribution of pathological subtypes are similar to those in other major salivary gland tumors. Other histological types are adenocarcinomas not otherwise specified (NOS), acinic cell carcinomas, polymorphous adenocarcinomas, basal cell adenocarcinomas and salivary ductal carcinoma which are reported to occur in the sublingual salivary gland (24).

In salivary gland cancer, histological grade is an important prognostic factor for survival outcome $(25,26)$. Therefore, confirming the histological grade is essential to management. The tumor grade of adenoid cystic carcinoma can be determined based on the proportion of solid component present (grade 1: no solid component, grade 2: equal to or less than $30 \%$ solid component, grade 3 : more than $30 \%$ solid component) $(21,26,27)$. Mucoepidermoid carcinoma and adenocarcinoma NOS were graded as low, intermediate and high grade, depending the tumor component, cell architecture, morphology and invasion characteristics (26). According to one article published recently, high-grade tumors were not uncommon (approximately 50\%), and pathological extraparenchymal extension was very frequent (80\%) (7).

\section{A prognostic staging system for malignant sublingual gland tumor}

Major salivary gland tumors (parotid, submandibular, and sublingual glands) have a separate prognostic staging in the current AJCC staging system (8th edition) $(28,29)$. Along with the criteria of tumor size, the presence of extra-parenchymal extension, tumor invasion to the overlying skin, mandible, ear canal, or facial nerve are anatomical landmarks for primary tumor $(\mathrm{T})$ classification. As previously stated, the sublingual glands are small (2-4 g) without a true surrounding capsule of the glands. Therefore, extra-parenchymal extension is very common in sublingual gland tumors (pT3: macroscopic or clinical extra-parenchymal extension), unless the tumor is very small in size. Notably, the tumor invasion of the lingual nerve is not the anatomical criteria for $\mathrm{T}$ classification, particularly in submandibular or sublingual gland tumors.

In contrast to sublingual gland tumors, tumors from the minor salivary glands follow a site-specific staging system (e.g., oral cavity, nasal cavity/paranasal sinus, or larynx).

\section{Treatment strategy for malignant sublingual gland tumors}

Safe surgical resection is the mainstay of treatment for malignant sublingual gland tumors when operable $(3,8,13,14,18,30)$. The extent and frequency of local invasion and regional spread highly depends on the tumor stage and pathological features, as in malignant salivary gland tumors.

Several considerations are required in surgery for sublingual gland tumors.

(I) Lingual nerve.

The treatment strategy for malignant sublingual gland tumors largely depends on histological subtype, tumor grade, tumor dimension, and anatomical relationship with adjacent structures. In 

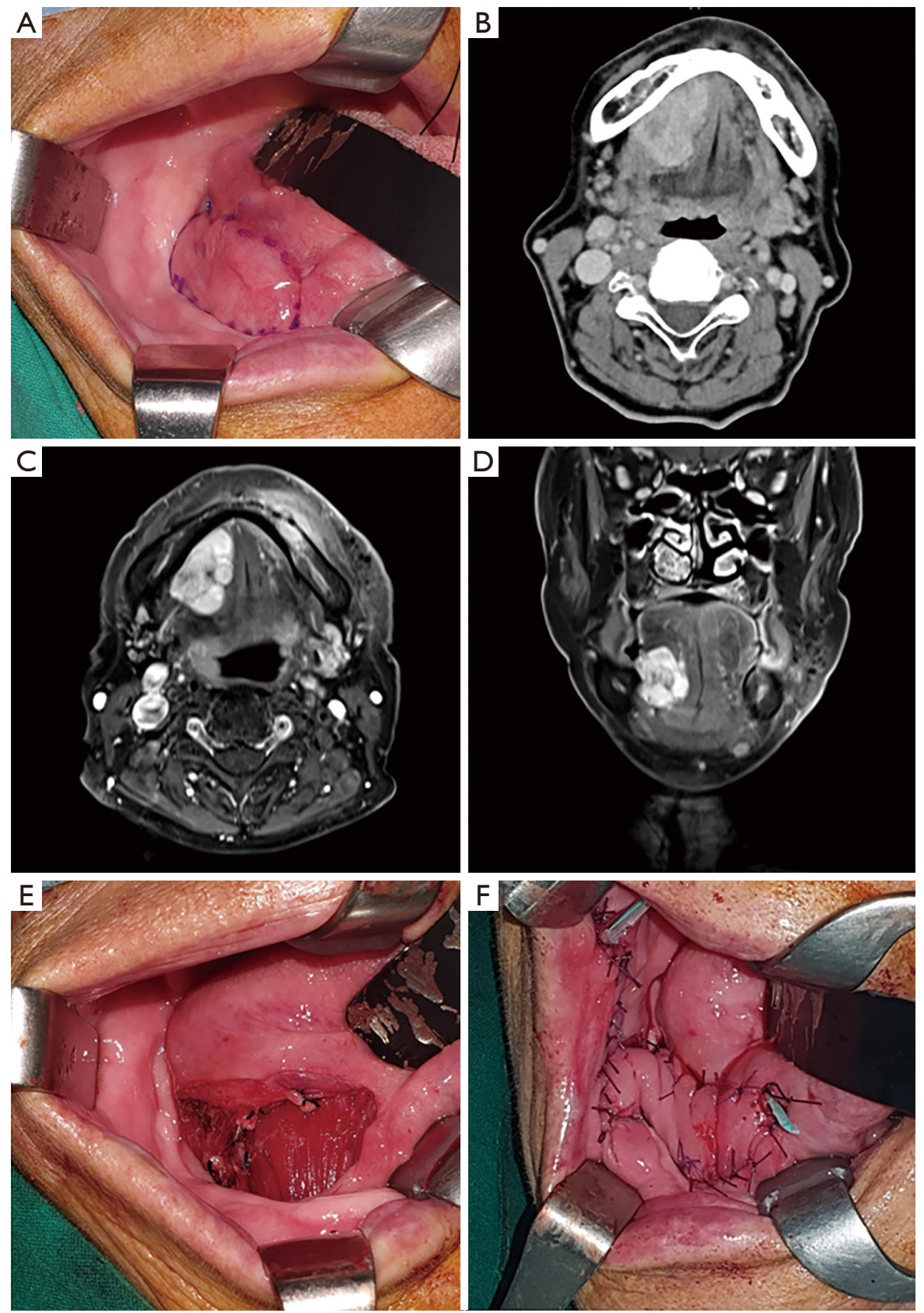

Figure 2 Representative images of a case with malignant sublingual gland tumor (Adenoid cystic carcinoma). (A) Submucosal mass in the floor of right mouth. (B) CT image, and (C,D) MRI images revealed the well-defined mass in the right sublingual space, abutting the mandible (without mandibular invasion). (E) Wide resection of the right sublingual tumor (with right neck dissection level I to III, resection of right submandibular gland). (F) Reconstruction of right floor of the mouth with a facial artery myomucosal flap.

cases of adenoid cystic carcinoma, most surgeons advocate the concurrent resection of the lingual nerve during curative surgery (range, $42.9 \%$ to $91.0 \%)(5,6,11)$. The rate of local invasion into the lingual nerve has been reported to range from $7.7 \%$ to $41.7 \%$ among surgical cases $(11,12,14)$. Conversely, the hypoglossal nerve appears to be safe from tumor invasion, because it is located 


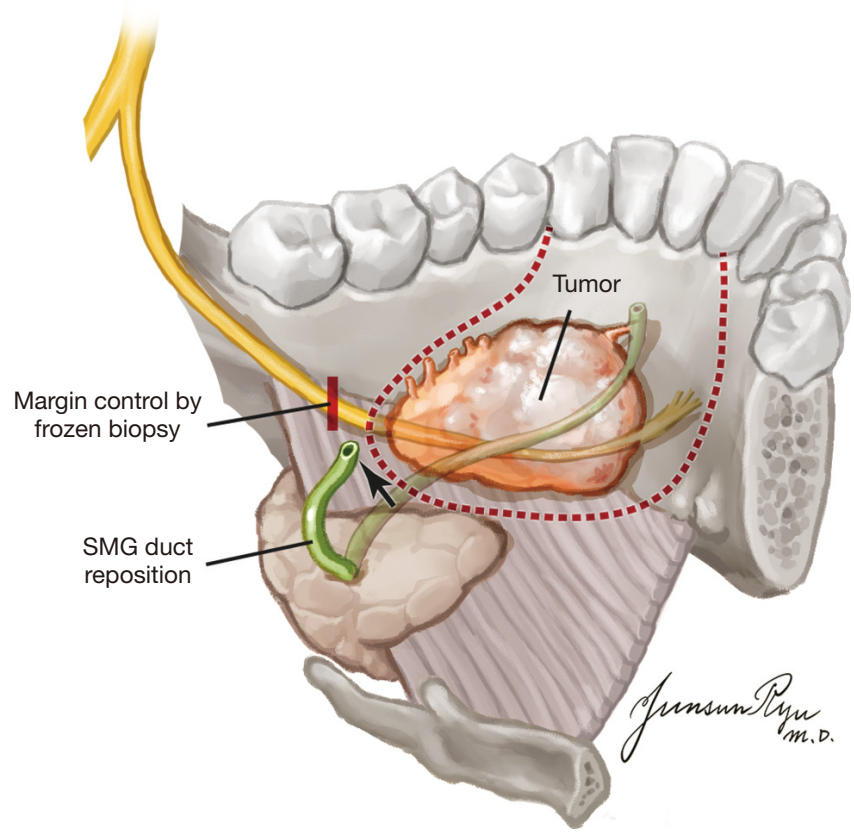

Figure 3 Rerouting of submandibular duct in surgery for small malignant sublingual gland tumors. In a case with small malignant sublingual gland tumor, the sublingual gland tumor can be resected safely without sacrifice of submandibular gland/duct system. The submandibular duct should be re-routed to the posterior part of the mouth floor, when the distal duct is resected en bloc with malignant sublingual gland tumor.

further away from most sublingual tumors (12).

In our series, the frequencies of lingual nerve invasion in adenoid cystic carcinoma and adenoid cystic carcinoma more than $4 \mathrm{~cm}$ in the largest dimension were $30.8 \%$ and $42.9 \%$, respectively (7).

(II) Submandibular gland and duct system.

Regarding the submandibular gland and ductal systems, submandibular gland excision in sublingual gland tumor surgery for a sufficient surgical safety margin was recommended in a previous study (8). When the sublingual gland tumors obstruct or invade the submandibular gland duct and the tumor dimension is small, the distal submandibular gland duct can be resected with the tumor, and the proximal portion of the duct is rerouted to the posterior oral cavity, preserving the submandibular gland (Figure 3) (31). However, if the tumor dimension is large (usually T3) and has high-grade pathology, a concurrent resection of the submandibular gland and duct appears to be oncologically safe.

(III) Neck lymph node management. Currently, the treatment guideline (the National Comprehensive Cancer Network Guidelines, https://www.nccn.org, Version: 2.2021, accessed on July 1,2021) recommends a prophylactic neck dissection for high-grade or T3-4 major salivary gland tumors.

In cases with clinical regional node metastasis, therapeutic ipsilateral neck dissection is a common clinical practice. The frequency of lymph node involvement in malignant sublingual gland tumors is not high (around 20\%); however, the frequency is over $50 \%$ in high-grade tumors (7), advocating prophylactic lymph node dissection at the time of primary tumor surgery.

The first regional spread to lymph nodes is confined to the neck lymph node levels Ib and IIa (7), and selective neck dissection frequently includes neck levels I to III for prophylactic treatment of neck lymph nodes in high-risk cases. In a subset of adenoid cystic carcinomas, the direct spread to the peri-tumor lymph node can occur (7). Therefore, these potential lymphatic basins should be evaluated or taken into consideration during treatment planning for malignant sublingual gland tumors.

(IV) Mandible.

The sublingual space is confined with the mandible, anterolaterally. If the sublingual tumors abut on the mandible, CT with a bone algorithm helps discriminate the cortical mandibular invasion. Meanwhile, bone marrow infiltration and perineural spread are best demonstrated on MRI. On CT images, the perineural spread is demonstrated as enlargement of the neural foramen. Tumors that arise from the sublingual gland (particularly, adenoid cystic carcinomas) may involve the inferior alveolar nerve, producing enlargement of the inferior alveolar nerve canal (22).

In case of mandible invasion, the involved mandible should be resected en bloc with the primary tumors, depending on the extent or length of the mandibular invasion (partial or segmental mandibulectomy). Bony reconstruction of the mandibular arch with fibular free flap is the first option for reconstruction.

(V) Reconstruction.

The surgical decision regarding reconstruction of 


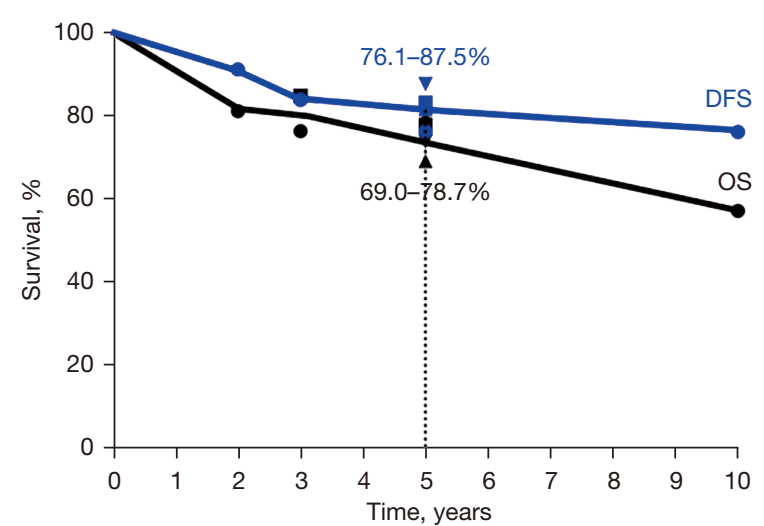

Figure 4 Overall and disease-free survivals of patients with malignant sublingual gland tumors. DFS, disease-free survival; OS, overall survival.

the mouth floor depends on the extent of resection or remaining tissues in the mouth floor. The two important structures supporting the mouth floor are the mylohyoid muscles (anteriorly) and the submandibular gland (posteriorly) (19). If these two structures are left in situ, the wound can be primarily closed or just skin-grafted. Even without them, the ventral aspect of tongue (medial margin of the defect) can be fixed to the mandible (lateral margin of the defect), with transmandibular horizontal mattress sutures (19).

However, some reconstruction plans should be considered, when transmandibular sutures are not possible due to the lack of soft tissues supporting the floor of the mouth or wide gap between the remaining tongue and the mandible. Local (facial artery-based myomucosal flap, submental flap), regional (supraclavicular flap, or pectoralis major flap), or free flaps (radial forearm or anterolateral thigh flaps) can prevent delayed wound healing, fistula formation between the oral cavity and the neck, and delay in the optimal adjuvant treatments.

\section{Adjuvant treatment}

According to the results of several previous reports, an advanced tumor stage correlates with poor prognosis, and adjuvant radiation therapy provides a survival benefit for patients with malignant sublingual gland tumors, who have risk factors $(1,3,11-13,18,20)$. The adverse features are intermediate or high-grade tumors, close or positive resection margins, neural/perineural invasion, lymph node metastases, lymphatic/vascular invasion, T3-4 tumors, and adenoid cystic carcinoma pathology $(18,32)$ (the National Comprehensive Cancer Network Guidelines, https://www. nccn.org, Version: 2.2021, accessed on July 1, 2021). For these tumors, adjuvant radiation is strongly recommended for better loco-regional control (level of evidence 2A).

Adjuvant concurrent chemo-radiotherapy or neoadjuvant chemotherapy has not been proven effective yet. Therefore, upfront surgery with or without adjuvant radiation therapy is the current standard of treatment for resectable malignant sublingual gland tumors. Approximately $10 \%$ of patients with malignant sublingual gland tumors are inoperable at diagnosis, and therapeutic options for them seem to be limited, only with chemotherapy or palliative local treatments (7).

\section{Outcomes and prognosis}

The malignant sublingual gland tumors frequently abut on the nearby structures in the narrow anatomical space; close surgical margin $(<5 \mathrm{~mm})$ is a common finding in the surgery of malignant sublingual gland tumors $(7,12,13)$. However, this cut-off value $(5 \mathrm{~mm})$ has not been proven reasonable for malignant sublingual gland tumors. Nevertheless, disease control appears relatively acceptable with surgery and adjuvant radiation treatments. Disease-free survival in surgical cases were $91.0 \%$ at 2 years, $83.7 \%$ at 3 years, $76.1-87.5 \%$ at 5 years and $76.0 \%$ at 10 years. Reported overall survival in all patients were $76.2-84.7 \%$ at 3 years, $69.0-78.7 \%$ at 5 years and $57.0 \%$ at 10 years $(3,7,12-14)$ (Figure 4). These findings may be explained by the relatively indolent tumor progression of salivary gland cancer and its good response to adjuvant radiation treatment (25). According to the published data $(3,6,7,11-14), 15-25 \%$ of cases recurred at 5 years after initial treatments. Most of the disease recurrences are lung or systemic metastases from adenoid cystic carcinoma pathology and local recurrences are rare with an adequate surgical resection and adjuvant radiation therapy.

However, the long-term outcomes and optimal surgical approach have not been adequately assessed until recently, because late or delayed recurrence can occur 5-15 years after the curative treatments similar to most salivary gland cancers (25). Thus, a large-scale follow-up study is necessary to confirm treatment outcomes of malignant sublingual gland tumors. 


\section{Conclusions}

Sublingual gland tumors are very rare, and most are malignant tumors. Treatment extent, clinical outcomes and prognosis of malignant sublingual gland tumors have not been well defined, due to the rarity of this disease. Herein, we presented the clinical characteristics and unique features of malignant sublingual gland tumors based on published reports.

Safe surgical resection is the mainstay of treatment for malignant sublingual gland tumors when operable; however, consensus has not been reached regarding the surgical approach and extent. Of note, it is reasonable to incorporate information about tumor characteristics (pathology and tumor grade) into the surgical decision, and the surgical approach should include the adequate management of neighboring structures. In addition, adjuvant radiation therapy provides a survival benefit for patients with malignant sublingual gland tumors, which have adverse features.

\section{Acknowledgments}

The artwork was created by one of the authors (J Ryu). The figures have never been published elsewhere.

Funding: This work was supported by the research fund of Samsung Medical Center (No. OTX0001031 and PHX0201451).

\section{Footnote}

Reporting Checklist: The authors have completed the Narrative Review reporting checklist. Available at https:// dx.doi.org/10.21037/gs-21-620

Conflicts of Interest: All authors have completed the ICMJE uniform disclosure form (available at https://dx.doi. org/10.21037/gs-21-620). The authors have no conflicts of interest to declare.

Ethical Statement: The authors are accountable for all aspects of the work in ensuring that questions related to the accuracy or integrity of any part of the work are appropriately investigated and resolved.

Open Access Statement: This is an Open Access article distributed in accordance with the Creative Commons Attribution-NonCommercial-NoDerivs 4.0 International
License (CC BY-NC-ND 4.0), which permits the noncommercial replication and distribution of the article with the strict proviso that no changes or edits are made and the original work is properly cited (including links to both the formal publication through the relevant DOI and the license). See: https://creativecommons.org/licenses/by-nc-nd/4.0/.

\section{References}

1. Andersen LJ, Therkildsen MH, Ockelmann HH, et al. Malignant epithelial tumors in the minor salivary glands, the submandibular gland, and the sublingual gland. Prognostic factors and treatment results. Cancer 1991;68:2431-7.

2. Carvalho AL, Nishimoto IN, Califano JA, et al. Trends in incidence and prognosis for head and neck cancer in the United States: a site-specific analysis of the SEER database. Int J Cancer 2005;114:806-16.

3. Lee RJ, Tong EL, Patel R, et al. Malignant sublingual gland tumors: demographics, prognostic factors, and treatment outcomes. Oral Surg Oral Med Oral Pathol Oral Radiol 2016;121:180-7.

4. Lukšić I, Virag M, Manojlović S, et al. Salivary gland tumours: 25 years of experience from a single institution in Croatia. J Craniomaxillofac Surg 2012;40:e75-81.

5. Sun G, Yang X, Tang E, et al. The treatment of sublingual gland tumours. Int J Oral Maxillofac Surg 2010;39:863-8.

6. Yu T, Gao QH, Wang XY, et al. Malignant sublingual gland tumors: a retrospective clinicopathologic study of 28 cases. Oncology 2007;72:39-44.

7. Park W, Park M, Choi K, et al. Analysis of local invasion and regional spread in malignant sublingual gland tumour: Implications for surgical planning. Int J Oral Maxillofac Surg 2021;50:1280-8.

8. Rinaldo A, Shaha AR, Pellitteri PK, et al. Management of malignant sublingual salivary gland tumors. Oral Oncol 2004;40:2-5.

9. Spiro RH, Armstrong J, Harrison L, et al. Carcinoma of major salivary glands. Recent trends. Arch Otolaryngol Head Neck Surg 1989;115:316-21.

10. Lin Y, Wang Y, Zhang H, et al. Sublingual Gland Tumors Worldwide: A Descriptive Retrospective Study of 839 Cases. J Oral Maxillofac Surg 2020;78:1546-56.

11. Andreasen S, Bjørndal K, Agander TK, et al. Tumors of the sublingual gland: a national clinicopathologic study of 29 cases. Eur Arch Otorhinolaryngol 2016;273:3847-56.

12. Kojima T, Hori R, Tanaka S, et al. A retrospective multicenter study of sublingual gland carcinoma in Japan. 
Auris Nasus Larynx 2020;47:111-5.

13. Liu Y, Li H, Qin L, et al. Prognostic Factors in Malignant Sublingual Salivary Gland Tumors. J Oral Maxillofac Surg 2017;75:1542-8.

14. Zdanowski R, Dias FL, Barbosa MM, et al. Sublingual gland tumors: clinical, pathologic, and therapeutic analysis of 13 patients treated in a single institution. Head Neck 2011;33:476-81.

15. Gontarz M, Urbańska-Gąsiorowska $M$, Bargiel J, et al. Sublingual gland neoplasms: clinicopathological study of 8 cases. Med Oral Patol Oral Cir Bucal 2021;26:e626-31.

16. Huang TT, Chou YF, Wen YH, et al. Resected tumours of the sublingual gland: 15 years' experience. Br J Oral Maxillofac Surg 2016;54:625-8.

17. Perez DE, Pires FR, Alves Fde A, et al. Sublingual salivary gland tumors: clinicopathologic study of six cases. Oral Surg Oral Med Oral Pathol Oral Radiol Endod 2005;100:449-53.

18. Bradley PJ, Ferris RL. Surgery for Malignant Sublingual and Minor Salivary Gland Neoplasms. Adv Otorhinolaryngol 2016;78:113-9.

19. Spiro RH. Treating tumors of the sublingual glands, including a useful technique for repair of the floor of the mouth after resection. Am J Surg 1995;170:457-60.

20. Lombardi D, McGurk M, Vander Poorten V, et al. Surgical treatment of salivary malignant tumors. Oral Oncol 2017;65:102-13.

21. Saito M, Nishiyama H, Maruyama S, et al. Adenoid cystic carcinoma of sublingual gland involving the submandibular duct. Dentomaxillofac Radiol 2008;37:421-4.

22. La'porte SJ, Juttla JK, Lingam RK. Imaging the floor of the mouth and the sublingual space. Radiographics 2011;31:1215-30.

23. Mukherji SK, Isaacs DL, Creager A, et al. CT detection of mandibular invasion by squamous cell carcinoma of the

Cite this article as: Park M, Cho J, Ryu J, Jeong HS. Diagnosis and management of malignant sublingual gland tumors: a narrative review. Gland Surg 2021;10(12):3415-3423. doi: $10.21037 / g s-21-620$ oral cavity. AJR Am J Roentgenol 2001;177:237-43.

24. Eveson JW, Cawson RA. Salivary gland tumours. A review of 2410 cases with particular reference to histological types, site, age and sex distribution. J Pathol 1985;146:51-8.

25. Park SI, Park W, Choi S, et al. Clinical Outcome of Minor Salivary Gland Cancers in the Oral Cavity: A Comparative Analysis With Squamous Cell Carcinomas of the Oral Cavity. Front Oncol 2020;10:881.

26. Seethala RR. An update on grading of salivary gland carcinomas. Head Neck Pathol 2009;3:69-77.

27. van Weert S, van der Waal I, Witte BI, et al. Histopathological grading of adenoid cystic carcinoma of the head and neck: analysis of currently used grading systems and proposal for a simplified grading scheme. Oral Oncol 2015;51:71-6.

28. Amin MB, Greene FL, Edge SB, et al. The Eighth Edition AJCC Cancer Staging Manual: Continuing to build a bridge from a population-based to a more "personalized" approach to cancer staging. CA Cancer J Clin 2017;67:93-9.

29. Amin MB, Edge SB, Greene FL, et al. AJCC cancer staging manual. 8th edition. Springer; 2017:95101. Available online: https://link.springer.com/ book/9783319406176

30. Jegadeesh N, Liu Y, Prabhu RS, et al. Outcomes and prognostic factors in modern era management of major salivary gland cancer. Oral Oncol 2015;51:770-7.

31. Sakakibara A, Minamikawa T, Hashikawa K, et al. Does salivary duct repositioning prevent complications after tumor resection or salivary gland surgery? J Oral Maxillofac Surg 2015;73:1003-7.

32. Colevas AD, Yom SS, Pfister DG, et al. NCCN Guidelines Insights: Head and Neck Cancers, Version 1.2018. J Natl Compr Canc Netw 2018;16:479-90. 